\title{
LOCAL $p$-SIDON SETS FOR LIE GROUPS
}

\author{
A. H. DOOLEY AND PAOLO M. SOARDI
}

ABSTRACT. It is shown that a compact Lie group admits no local $p$-Sidon sets of unbounded degree.

Let $G$ be a compact group, and let $1 \leqslant p<2$. A subset $R$ of the dual of $G$ is called a local $p$-Sidon set if there exists a constant $B$ such that for every $\sigma \in R$ and for every $d_{\sigma} \times d_{\sigma}$ matrix $A_{\sigma}$,

$$
\left\|A_{\sigma}\right\|_{p} \leqslant B d_{\sigma}^{1 / p^{\prime}}\left\|\operatorname{tr} A_{\sigma} \sigma(\cdot)\right\|_{\infty} .
$$

THEOREM. If $G$ is a compact Lie group, and if $R$ is a local p-Sidon set for $G$, then $\sup \left\{d_{\sigma} \mid \sigma \in R\right\}<\infty$.

Proof. We first note that, if $G$ is an arbitrary compact group, $R$ is a $p$-Sidon set for $G$, and if $r>1$, then there exists a constant $\kappa_{r}$ such that for all $\sigma \in R$

$$
\left\|\chi_{\sigma}\right\|_{r} \leqslant \kappa_{r} d_{\sigma}^{2 / p^{\prime}}
$$

where $\chi_{\sigma}(x)=\operatorname{tr}(\sigma(x))$.

To see this, we first use a simple duality argument to see that (1) is equivalent to: there exists a constant $C$ such that for every $\sigma \in R$ and for every $d_{\sigma} \times d_{\sigma}$ matrix $A_{\sigma}$, there exists $g \in L^{1}(G)$ such that $\hat{g}(\sigma)=A_{\sigma}$, and $\|g\|_{1} \leqslant C d_{\sigma}^{1 / p^{\prime}}\left\|A_{\sigma}\right\|_{p^{\prime}}$. Thus for every $\sigma \in R$ and for every $d_{\sigma} \times d_{\sigma}$ unitary matrix $W$, there exists $g_{W} \in L^{1}(G)$ with $\hat{g}_{W}=W^{*}$, and $\left\|g_{W}\right\|_{1} \leqslant$ $C d_{\sigma}^{1 / p^{\prime}}\left\|W^{*}\right\|_{p^{\prime}}=d_{\sigma}^{2 / p^{\prime}}$. Since $\chi_{\sigma}=g_{W} *(\operatorname{tr}(W \cdot \sigma(\cdot)))$ we have

$$
\begin{aligned}
\left\|\chi_{\sigma}\right\|_{r} & \leqslant\left\|g_{W}\right\|_{1}\left(\int_{G}|\operatorname{tr}(W \cdot \sigma(x))|^{r} d x\right)^{1 / r} \\
& \leqslant C d_{\sigma}^{2 / p^{\prime}}\left(\int_{G}|\operatorname{tr}(W \cdot \sigma(x))|^{r} d x\right)^{1 / r} .
\end{aligned}
$$

Hence, integrating over the $d_{\sigma} \times d_{\sigma}$ unitary group, $\mathcal{U}\left(d_{\sigma}\right)$ with respect to normalized Haar measure $d W$, and using Hölder's inequality, we obtain

$$
\begin{aligned}
\left\|\chi_{\sigma}\right\|_{r} & \leqslant C d_{\sigma}^{2 / p^{\prime}}\left(\int_{G} \int_{Q_{\left(d_{\sigma}\right)}}|\operatorname{tr}(W \cdot \sigma(x))|^{r} d W d x\right)^{1 / r} \\
& =C d_{\sigma}^{2 / p^{\prime}}\left(\int_{U_{\left(d_{\sigma}\right)}}|\operatorname{tr} W|^{r} d W\right)^{1 / r} .
\end{aligned}
$$

Received by the editors February 7, 1978.

AMS (MOS) subject classifications (1970). Primary 42A44, 43A14.

(c) American Mathematical Society 1978 
The last equality follows from the translation invariance of $d W$. It is easily established, however (cf. [2, (29.12)]), that there exists a bound $K_{r}$, independent of $d_{\sigma}$, for $\left(\int_{थ\left(d_{\sigma}\right)}|\operatorname{tr} W|^{r} d W\right)^{1 / r}$. Thus we obtain (2).

Suppose now that $G$ is a compact Lie group. In [1, Theorem (5.4)], the following estimate is given for the $r$-norms of the irreducible characters; let $M_{G} \in \mathbf{R}$ be as in [1, (5.5)]. Then for $r>M_{G}$, there exists a constant $\kappa_{r}$ such that

$$
\kappa_{r} d_{\sigma}^{1-M_{G} / r} \leqslant\left\|\chi_{\sigma}\right\|_{r} .
$$

From (2) and (3), it follows that, for all $r>M_{G}$, $\sup _{\sigma \in R} d_{\sigma}^{1-2 / p^{\prime}-M_{G} / r}<\infty$, and hence, since $p<2, \sup _{\sigma \in R} d_{\sigma}<\infty$.

It follows that, if $G$ is a compact semisimple Lie group, $G$ has no infinite local $p$-Sidon sets.

It should be noted that a set $R$ with $\sup \left\{d_{\sigma} \mid \sigma \in R\right\}<\infty$ is local Sidon and hence local $p$-Sidon for all $p[3]$.

\section{REFERENCES}

1. A. H. Dooley, Norms of characters and lacunarity for compact Lie groups, J. Functional Analysis (to appear).

2. E. Hewitt and K. A. Ross, Abstract harmonic analysis, vol. 2, Springer-Verlag, Berlin, 1970.

3. J. F. Price, Local Sidon sets and uniform convergence of Fourier series, Israel J. Math. 17 (1974), 169-175.

4. P. M. Soardi, $\delta \vartheta_{2}$ has no infinite local p-Sidon sets (preprint).

School of Mathematical Sciences, The flinders University of South Australia, Bedford Park, S.A., 5042, Australia

Istituto Matematico dell'Universitì, via Saldini 50, 20133 Milano, Italy 